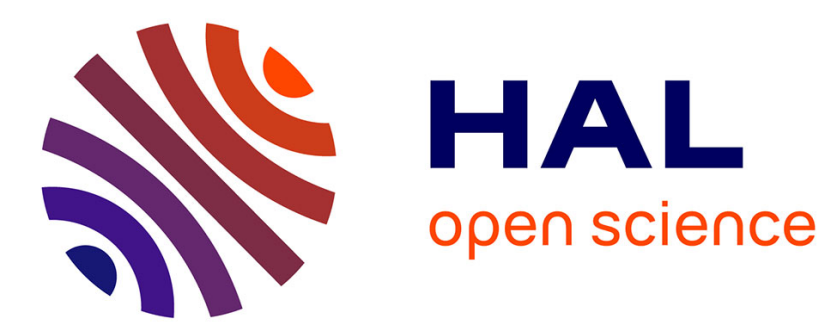

\title{
Output Feedback Robust Hinf Control of Uncertain Active Fault Tolerant Control Systems via Convex Analysis
}

Samir Aberkane, Dominique Sauter, Jean-Christophe Ponsart

\section{- To cite this version:}

Samir Aberkane, Dominique Sauter, Jean-Christophe Ponsart. Output Feedback Robust Hinf Control of Uncertain Active Fault Tolerant Control Systems via Convex Analysis. International Journal of Control, 2007, 81 (2), pp.252-263. 10.1080/00207170701535959 . hal-00201091

\section{HAL Id: hal-00201091 \\ https://hal.science/hal-00201091}

Submitted on 23 Dec 2007

HAL is a multi-disciplinary open access archive for the deposit and dissemination of scientific research documents, whether they are published or not. The documents may come from teaching and research institutions in France or abroad, or from public or private research centers.
L'archive ouverte pluridisciplinaire HAL, est destinée au dépôt et à la diffusion de documents scientifiques de niveau recherche, publiés ou non, émanant des établissements d'enseignement et de recherche français ou étrangers, des laboratoires publics ou privés. 


\title{
Output Feedback Robust $\mathcal{H}_{\infty}$ Control of Uncertain Active Fault Tolerant Control Systems via Convex Analysis
}

\author{
S. Aberkane* D. Sauter and J. C. Ponsart \\ CRAN - CNRS UMR 7039 \\ Université Henri Poincaré, Nancy 1, BP 239, \\ F-54506 Vandœuvre-lès-Nancy Cedex
}

\begin{abstract}
This paper deals with the problem of $\mathcal{H}_{\infty}$ and robust $\mathcal{H}_{\infty}$ control, via dynamic output feedback, of continuous time Active Fault Tolerant Control Systems with Markovian Parameters (AFTCSMP) subject to both structured and unstructured parameter uncertainties. The above problematic is addressed under a convex programming approach. Indeed, the fundamental tool in the analysis is an LMI (Linear Matrix Inequalities) characterization of dynamical compensators that stochastically (robustly) stabilize the closed loop system and ensure $\mathcal{H}_{\infty}$ and robust $\mathcal{H}_{\infty}$ performances. Numerical examples are presented to illustrate the theoretical results.
\end{abstract}

\section{Introduction}

Modern technological systems, such as nuclear power plants, aircrafts and space stations, rely on sophisticated control functions in order to achieve high levels of reliability and performances. For these safety-critical systems, Fault Tolerant Control Systems (FTCS) have been developed to meet these essential objectives. FTCS have been a subject of great practical importance, which has attracted a lot of interest for the last three decades. A review on reconfigurable fault tolerant control systems can be found in (Blanke et al. 2003, Zhang et al. 2003).

Active fault tolerant control systems are feedback control systems that reconfigure the control law in real time based on the response from an automatic fault detection and identification (FDI) scheme. The dynamic behaviour of active fault tolerant control systems (AFTCS) is governed by stochastic differential equations (because the failures and failure detection occur randomly) and can be viewed as a general hybrid system (Srichander et al. 1993). A major class of hybrid systems is jump linear systems (JLS). In JLS, a single jump process is used to describe the random variations affecting the system parameters. This process is represented by a finite state Markov chain and is called the plant regime mode. The theory of stability, optimal control and $\mathcal{H}_{2} / \mathcal{H}_{\infty}$ control, as well as important applications of such systems, can be found in several papers in the current literature, for instance in (Boukas et al. 1999, 2002, 2004, 2005, 2006, Costa et al. 1999, de Farias et al. 2000, de Souza et al. 1993, Ji et al. 1990, 1992).

To deal with AFTCS, another class of hybrid systems was defined, denoted as active fault tolerant control systems with Markovian parameters (AFTCSMP). In this class of hybrid systems, two random processes are defined: the first random process represents system components failures and the second random process represents the FDI process used to reconfigure the control law. This model was proposed by Srichander and Walker (Srichander et al. 1993). Necessary and sufficient conditions for stochastic stability of AFTCSMP were developed for a single component failure (actuator failures). In (Mahmoud et al. 1999), the authors proposed a dynamical model that takes into account multiple failures occurring at different locations in the system, such as in control actuators and plant components. The authors derived necessary and sufficient conditions for the stochastic stability in the mean square sense. The problem of stochastic stability of

\footnotetext{
*Corresponding author. Email: samir.aberkane@cran.uhp-nancy.fr
} 
AFTCSMP in the presence of noise, parameter uncertainties, detection errors, detection delays and actuator saturation limits has also been investigated in (Mahmoud et al. 1999, 2001, 2002, 2003). Another issue related to the synthesis of fault tolerant control laws was also addressed by (Mahmoud et al. 2000, Shi et al. 1997, 2003). In (Mahmoud et al. 2000), the authors designed an optimal control law for AFTCSMP using the matrix minimum principle to minimize an equivalent deterministic cost function. The problem of $\mathcal{H}_{\infty}$ and robust $\mathcal{H}_{\infty}$ control (in the presence of norm bounded parameter uncertainties) was treated in (Shi et al. 1997, 2003) for both continuous and discrete time AFTCSMP. The authors defined a single failure process to characterize random failures affecting the system (i.e. the location and the nature of the faulty components were not taken into account), and they showed that the state feedback control problem can be solved in terms of the solutions of a set of coupled Riccati inequalities. However, to the best of author's knowledge, the problem of dynamic output feedback $\mathcal{H}_{\infty}$ and robust $\mathcal{H}_{\infty}$ control of an uncertain AFTCSMP (in the case of structured and unstructured uncertainties, respectively) subject to multiple failures processes has not been fully investigated.

In this paper, we are concerned with the problem of dynamic output feedback $\mathcal{H}_{\infty}$ and robust $\mathcal{H}_{\infty}$ control of an uncertain AFTCSMP via convex analysis, which has shown to be a powerful tool to derive numerical algorithms for several important control problems. The first problematic we consider in this paper is the dynamic output feedback stochastic stabilization and robust stochastic stabilization of uncertain AFTCSMP subject to multiple failure processes: one for plant components and the other for actuators. The main reason for using two independent failure processes is that it allows the modelling of faults at different locations with independent failure characteristics (Mahmoud et al. 2003). The uncertainties we consider here are modeled in three different forms: norm bounded uncertainties, linear combination uncertainties and value bounded uncertainties. The motivation to consider the above three different uncertainty forms is to provide a more unified treatment to handle both structured and unstructured parameter uncertainties. It is shown that the necessary and sufficient conditions for the internal exponential stability in the mean square sense (robust internal exponential stability, respectively) can be written in terms of an LMI feasibility problem. This leads to an LMI characterization of all dynamical output feedback compensators that satisfied the stability requirements. Having obtained this result, we can move on the control problems and write the dynamic output feedback $\mathcal{H}_{\infty}$ and robust $\mathcal{H}_{\infty}$ control problems of continuous time uncertain AFTCSMP in terms of LMI optimization problems. The convex approach naturally leads to powerful numerical algorithms to solve these problematic.

This paper is organized as follows: section 2 describes the dynamical model of the system with appropriately defined random processes. A brief summary of basic stochastic terms, results and definitions are given in section 3. Section 4 derives the necessary and sufficient conditions for the stochastic (robust) exponential stability in the mean square sense, and the LMI characterization of the dynamical compensators. Sections 5 and 6 consider, respectively, the $\mathcal{H}_{\infty}$ and robust $\mathcal{H}_{\infty}$ control problems for the output feedback system via LMI optimization problems. Finally, a conclusion is given in section 7 .

Notations. The notations in this paper are quite standard. $\mathbb{R}^{m \times n}$ is the set of $m$-by- $n$ real matrices. $A^{T}$ is the transpose of the matrix $A$. The notation $X \geq Y(X>Y$, respectively), where $X$ and $Y$ are symmetric matrices, means that $X-Y$ is positive semi-definite (positive definite, respectively); $\square$ and $\mathbf{0}$ are identity and zero matrices of appropriate dimensions, respectively; $\mathcal{E}\{\cdot\}$ denotes the expectation operator with respect to some probability measure $P ; L^{2}[0, \infty)$ stands for the space of square-integrable vector functions over the interval $[0, \infty) ;\|\cdot\|$ refers to either the Euclidean vector norm or the matrix norm, which is the operator norm induced by the standard vector norm; $\|\cdot\|_{2}$ stands for the norm in $L^{2}[0, \infty)$; while $\|\cdot\|_{\mathcal{E}_{2}}$ denotes the norm in $L^{2}((\Omega, \mathcal{F}, P),[0, \infty)) ;(\Omega, \mathcal{F}, P)$ is a probability space. In block matrices, $\star$ indicates symmetric terms: $\left[\begin{array}{cc}A & B \\ B^{\prime} & C\end{array}\right]=\left[\begin{array}{cc}A & \star \\ B^{\prime} & C\end{array}\right]=\left[\begin{array}{cc}A & B \\ \star & C\end{array}\right]$.

\section{Dynamical Model of AFTCSMP with Parameter Uncertainties}

To describe the class of linear systems with Markovian jumping parameters that we deal with in this paper, let us fix a complete probability space $(\Omega, \mathcal{F}, P)$. This class of systems owns a hybrid state vector. The first component vector is continuous and represents the system states, and the second one is discrete and 
represents the failure processes affecting the system. The dynamical model of the AFTCSMP defined in the fundamental probability space $(\Omega, \mathcal{F}, P)$, is described by the following differential equations:

$$
\varphi:\left\{\begin{array}{l}
\dot{x}(t)=[A(\xi(t))+\Delta A(\xi(t))] x(t)+B(\eta(t)) u(y(t), \psi(t), t)+E(\xi(t), \eta(t)) w(t) \\
y(t)=C_{2} x(t)+D_{2}(\xi(t), \eta(t)) w(t) \\
z_{\infty}(t)=C_{1} x(t)+D_{1}(\eta(t)) u(y(t), \psi(t), t)
\end{array}\right.
$$

where $x(t) \in \mathbb{R}^{n}$ is the system state, $u(y(t), \psi(t), t) \in \mathbb{R}^{r}$ is the system input, $y(t) \in \mathbb{R}^{q}$ is the system measured output, $z(t) \in \mathbb{R}^{p}$ is the controlled output, $w(t) \in \mathbb{R}^{m}$ is the disturbance input which belongs to $L^{2}[0, \infty)$ and $\Delta A(\xi(t))$ is a real, time-varying matrix function representing the system uncertainties, $\xi(t), \eta(t)$ and $\psi(t)$ represent the plant component failure process, the actuator failure process and the FDI process, respectively. $\xi(t), \eta(t)$ and $\psi(t)$ are separable and measurable Markov processes with finite state spaces $Z=\{1,2, \ldots, z\}, S=\{1,2, \ldots, s\}$ and $R=\{1,2, \ldots, r\}$, respectively. The matrices $A(\xi(t)), B(\eta(t))$, $E(\xi(t), \eta(t)), D_{2}(\xi(t), \eta(t)), D_{1}(\eta(t))$ and $\Delta A(\xi(t))$ are properly dimensioned matrices which depend on random parameters.

We introduce a full order dynamic output feedback compensator $\left(\varphi_{c}\right)$ of the form:

$$
\varphi_{c}:\left\{\begin{array}{l}
\dot{v}(t)=A_{c}(\psi(t)) v(t)+B_{c}(\psi(t)) y(t) \\
u(t)=C_{c}(\psi(t)) v(t)
\end{array}\right.
$$

where $A_{c}(\psi(t)) \in \mathbb{R}^{n \times n}, B_{c}(\psi(t)) \in \mathbb{R}^{n \times q}, C_{c}(\psi(t)) \in \mathbb{R}^{r \times n}$. Applying the controller $\varphi_{c}$ to the uncertain $\operatorname{AFTCSMP} \varphi$, we obtain the following closed loop system:

$$
\left\{\begin{array}{l}
\dot{\chi}(t)=[\Lambda(\xi(t), \eta(t), \psi(t))+\Delta \Lambda(\xi(t))] \chi(t)+\bar{E}(\xi(t), \eta(t), \psi(t)) w(t) \\
\bar{y}(t)=\bar{C}_{2}(\psi(t)) \chi(t)+\bar{D}_{2}(\xi(t), \eta(t)) w(t) \\
z_{\infty}(t)=\bar{C}_{1}(\eta(t), \psi(t)) \chi(t)
\end{array}\right.
$$

where:

$$
\begin{aligned}
& \chi(t)=\left[x(t)^{T}, v(t)^{T}\right]^{T} ; \bar{y}(t)=\left[y(t)^{T}, u(t)^{T}\right]^{T} ; \Lambda(\xi(t), \eta(t), \psi(t))=\left[\begin{array}{cc}
A(\xi(t)) & B(\eta(t)) C_{c}(\psi(t)) \\
B_{c}(\psi(t)) C_{2} & A_{c}(\psi(t))
\end{array}\right] ; \\
& \Delta \Lambda(\xi(t))=\left[\begin{array}{cr}
\Delta A(\xi(t)) & \mathbf{0} \\
\mathbf{0} & \mathbf{0}
\end{array}\right] ; \bar{E}(\xi(t), \eta(t), \psi(t))=\left[\begin{array}{c}
E(\xi(t), \eta(t)) \\
B_{c}(\psi(t)) D_{2}(\xi(t), \eta(t))
\end{array}\right] ; \\
& \bar{C}_{2}(\psi(t))=\left[\begin{array}{cc}
C_{2} & \mathbf{0} \\
\mathbf{0} & C_{c}(\psi(t))
\end{array}\right] ; \bar{D}_{2}(\xi(t), \eta(t))=\left[\begin{array}{c}
D_{2}(\xi(t), \eta(t)) \\
\mathbf{0}
\end{array}\right] ; \\
& \bar{C}_{1}(\eta(t), \psi(t))=\left[\begin{array}{ll}
C_{1} & \left.D_{1}(\eta(t)) C_{c}(\psi(t))\right] .
\end{array}\right.
\end{aligned}
$$

\subsection{The FDI and the Failure Processes}

$\xi(t), \eta(t)$ and $\psi(t)$ being homogeneous Markov processes with finite state spaces, we can define the transition probability of the plant components failure process as (Mahmoud et al. 2003, Srichander et al. 1993):

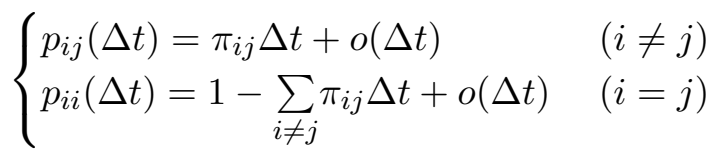


The transition probability of the actuator failure process is given by:

$$
\begin{cases}p_{k l}(\Delta t)=\nu_{k l} \Delta t+o(\Delta t) & (k \neq l) \\ p_{k k}(\Delta t)=1-\sum_{k \neq l} \nu_{k l} \Delta t+o(\Delta t) & (k=l)\end{cases}
$$

where $\pi_{i j}$ is the plant components failure rate, and $\nu_{k l}$ is the actuator failure rate.

Given that $\xi=k$ and $\eta=l$, the conditional transition probability of the FDI process $\psi(t)$ is:

$$
\begin{cases}p_{i v}^{k l}(\Delta t)=\lambda_{i v}^{k l} \Delta t+o(\Delta t) & (i \neq v) \\ p_{i i}^{k l}(\Delta t)=1-\sum_{i \neq v} \lambda_{i v}^{k l} \Delta t+o(\Delta t) & (i=v)\end{cases}
$$

Here, $\lambda_{i v}^{k l}$ represents the transition rate from $i$ to $v$ for the Markov process $\psi(t)$ conditioned on $\xi=k \in Z$ and $\eta=l \in S$. Depending on the values of $i, v \in R, k \in Z$ and $l \in S$, various interpretations, such as rate of false detection and isolation, rate of correct detection and isolation, false alarm recovery rate, etc, can be given to $\lambda_{i v}^{k l}$ (Mahmoud et al. 2003, Srichander et al. 1993).

Remark 1 Practically, it is difficult to determine the exact values of the conditional transition rates $\lambda_{i v}^{k l}$. However, Monte Carlo simulations and prior information can be used to approximate these rates (Mahmoud et al. 2003).

\subsection{The Model of Parameter Uncertainties}

The different plant models used in robust control system can have their own type of uncertainty representation. In general, modeling uncertainty is classified in a number of different ways (Zhou et al. 1996). In this work, we consider the following forms of parameter uncertainties (Boukas et al. 1999, Mahmoud et al. 2000, Zhou et al. 1996):

2.2.1 Structured Uncertainties. This type of uncertainties arise from the linearization of a non linear system around a fixed operating point. Two classes of structured uncertainties will be studied.

1) Norm Bounded Uncertainty (NBU)

The admissible parameter uncertainties are modelled as:

$$
\Delta A(\xi(t))=H(\xi(t)) F(\xi(t)) G(\xi(t))
$$

where $H(\xi(t)) \in \mathbb{R}^{n \times p_{u}}, F(\xi(t)) \in \mathbb{R}^{p_{u} \times q_{u}}$ and $G(\xi(t)) \in \mathbb{R}^{q_{u} \times n} \cdot H(\xi(t)), G(\xi(t))$ are known constant matrices, and $F(\xi(t))$ is a Lipschitz measurable matrix function satisfying the condition:

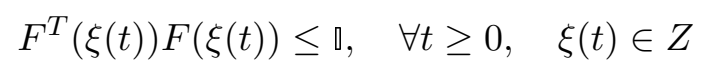

\section{2) Linear Combination Uncertainty (LCU)}

This form of structured uncertainty is modeled as:

$$
\Delta A(\xi(t))=\sum_{l=1}^{p_{\Delta}} N_{l}(\xi(t)) a_{l}(\xi(t), t)
$$

where $N_{l}(\xi(t))$ are known matrices and $a_{l}(t)$ represent the bounded uncertain parameters, i.e.

$$
\left|a_{l i}(t)\right| \leq \bar{a}_{i} \quad \forall l \in\left\{1, \ldots, p_{\Delta}\right\}, \quad \xi(t)=i \in Z, \quad \forall t \geq 0
$$


where $\bar{a}_{i}$ are given positive scalars. The matrix $N_{l}(\xi(t))$ can be written as:

$$
N_{l}(\xi(t))=d_{l}(\xi(t)) v_{l}^{T}(\xi(t))
$$

where $d_{l}(\xi(t))$ and $v_{l}(\xi(t))$ are matrices of appropriate dimensions.

2.2.2 Unstructured Uncertainties. The unstructured uncertainties are associated with unmodeled dynamics, truncation of high frequency modes, or nonlinearity in the system. A major class of this type of uncertainty is the Value Bounded Uncertainty (VBU). In this class, parameter uncertainties are modeled as:

$$
\left|\Delta A_{i}\right| \leq M_{i}, \quad M_{i}=\left[m_{l j}\right]_{i} ; m_{l j} \geq 0
$$

where $|\cdot|$ represents the modulus of entries of the corresponding matrix and $M_{i}$ is known constant matrix with all positive elements.

For notational simplicity, we will denote $A(\xi(t))=A_{i}, H(\xi(t))=H_{i}, F(\xi(t))=F_{i}$ and $G(\xi(t))=G_{i}$ when $\xi(t)=i \in Z, B(\eta(t))=B_{j}$ and $D_{1}(\eta(t))=D_{1 j}$ when $\eta(t)=j \in S, E(\xi(t), \eta(t))=E_{i j}$ and $D_{2}(\xi(t), \eta(t))=D_{2 i j}$ when $\xi(t)=i \in Z, \eta(t)=j \in S$ and $A_{c}(\psi(t))=A_{c k}, B_{c}(\psi(t))=B_{c k}, C_{c}(\psi(t))=C_{c k}$ when $\psi(t)=k \in R$. We also denote $x(t)=x_{t}, y(t)=y_{t}, z_{\infty}(t)=z_{\infty t}, w(t)=w_{t}, \xi(t)=\xi_{t}, \eta(t)=\eta_{t}$, $\psi(t)=\psi_{t}$ and the initial conditions $x\left(t_{0}\right)=x_{0}, \xi\left(t_{0}\right)=\xi_{0}, \eta\left(t_{0}\right)=\eta_{0}$ and $\psi\left(t_{0}\right)=\psi_{0}$.

\section{Definitions and Basic Results}

For system (1), when $u_{t} \equiv 0$ and $\Delta A\left(\xi_{t}\right) \equiv 0$ for all $t \geq 0$, we have the following definition:

DeFINITION 3.1 System (1) is said to be internally exponentially stable in the mean square sense (IESS), if there exist positive constants $\alpha$ and $\beta$ such that the solution of

$$
\dot{x}_{t}=A\left(\xi_{t}\right) x_{t}
$$

satisfies the following inequality

$$
\mathcal{E}\left\{\left\|x_{t}\right\|^{2}\right\} \leq \beta\left\|x_{0}\right\|^{2} \exp \left[-\alpha\left(t-t_{0}\right)\right]
$$

for arbitrary initial conditions $\left(x_{0}, \xi_{0}, \eta_{0}, \psi_{0}\right)$.

When the system uncertainties are not equal to zero, the concept of stochastic stability becomes robust stochastic stability and is defined for system (1) as follows:

Definition 3.2 System (1) is said to be robustly internally exponentially stable in the mean square sense (RIESS), if there exist positive constants $\alpha$ and $\beta$ such that the solution of

$$
\dot{x}_{t}=\left[A\left(\xi_{t}\right)+\Delta A\left(\xi_{t}\right)\right] x_{t}
$$

satisfies (4) for arbitrary initial conditions $\left(x_{0}, \xi_{0}, \eta_{0}, \psi_{0}\right)$ and for all admissible uncertainties.

We conclude this section by recalling the following lemmas which will be useful for the proof of our main results in the next sections.

Lemma 3.3 (Petersen et al. 1987) Let $G, M, N$ be real matrices of appropriate dimensions. Then, for any

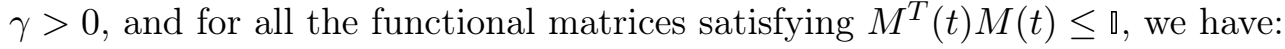

$$
2 x^{T} P G M(t) N x \leq \gamma x^{T} P G G^{T} P x+(1 / \gamma) x^{T} N^{T} N x
$$


Lemma 3.4 (Schur complement) (Boyd et al. 1994) Given constant matrices $M, L, Q$ of appropriate dimensions where $M$ and $Q$ are symmetric, then $Q>0$ and $M+L^{T} Q^{-1} L<0$ if and only if:

$$
\left[\begin{array}{cc}
M & L^{T} \\
\star & -Q
\end{array}\right]<0
$$

\section{Stochastic Stabilization}

In this section, we will first derive a necessary and sufficient condition for the internal exponential (robust exponential) stability in the mean square sense of the system (3), in term of coupled matrix inequalities, and then we will give an LMI characterization of dynamical compensators $\left(\varphi_{c}\right)$ that internally exponentially stabilize (robustly stabilize) the closed-loop system in the mean square sense.

\subsection{Internal Stochastic Stability}

We are concerned by the problem of finding all the dynamical compensators $\varphi_{c}$, as defined in section 2 , such that the AFTCSMP (3) becomes internally exponentially stable in the mean square sense.

The following proposition gives a necessary and sufficient condition for internal exponential stability in the mean square sense for the system (3).

Proposition 4.1 A necessary and sufficient condition for IESS of the system (3) is that there exist symmetric positive-definite matrices $P_{i j k}, i \in Z, j \in S$ and $k \in R$ such that:

$$
\tilde{\Lambda}_{i j k}^{T} P_{i j k}+P_{i j k} \tilde{\Lambda}_{i j k}+\sum_{\substack{h \in Z \\ h \neq i}} \pi_{i h} P_{h j k}+\sum_{\substack{l \in S \\ l \neq j}} \nu_{j l} P_{i l k}+\sum_{\substack{v \in R \\ v \neq k}} \lambda_{k v}^{i j} P_{i j v}=\Xi_{i j k}<0
$$

where: $\tilde{\Lambda}_{i j k}=\Lambda_{i j k}-0.5 \square\left(\sum_{\substack{h \in Z \\ h \neq i}} \pi_{i h}+\sum_{\substack{l \in S \\ l \neq j}} \nu_{j l}+\sum_{\substack{v \in R \\ v \neq k}} \lambda_{k v}^{i j}\right)$.

Proof See (Aberkane et al. 2005).

We are now able to present the following proposition which gives an LMI characterization of dynamical compensators $\left(\varphi_{c}\right)$ that internally stabilize the closed-loop system in the mean square sense.

Proposition 4.2 A necessary and sufficient condition for IESS of the system (3) is that the following matrix inequalities:

$$
\begin{gathered}
{\left[\begin{array}{rr}
\tilde{A}_{i j k} Y_{i j k}+Y_{i j k} \tilde{A}_{i j k}^{T}+F_{i j k}^{T} B_{j}^{T}+B_{j} F_{i j k} & R_{i j k}(Y) \\
\star & S_{i j k}(Y)
\end{array}\right]<0, \quad\left[\begin{array}{cc}
Y_{i j k} & 0 \\
\star & X_{i j k}
\end{array}\right]>0} \\
\tilde{A}_{i j k}^{T} X_{i j k}+X_{i j k} \tilde{A}_{i j k}+C_{2}^{T} H_{i j k}^{T}+H_{i j k} C_{2}+\sum_{\substack{h \in Z \\
h \neq i}} \pi_{i h} X_{h j k}+\sum_{\substack{l \in S \\
l \neq j}} \nu_{j l} X_{i l k}+\sum_{\substack{v \in R \\
v \neq k}} \lambda_{k v}^{i j} X_{i j v}<0
\end{gathered}
$$


where

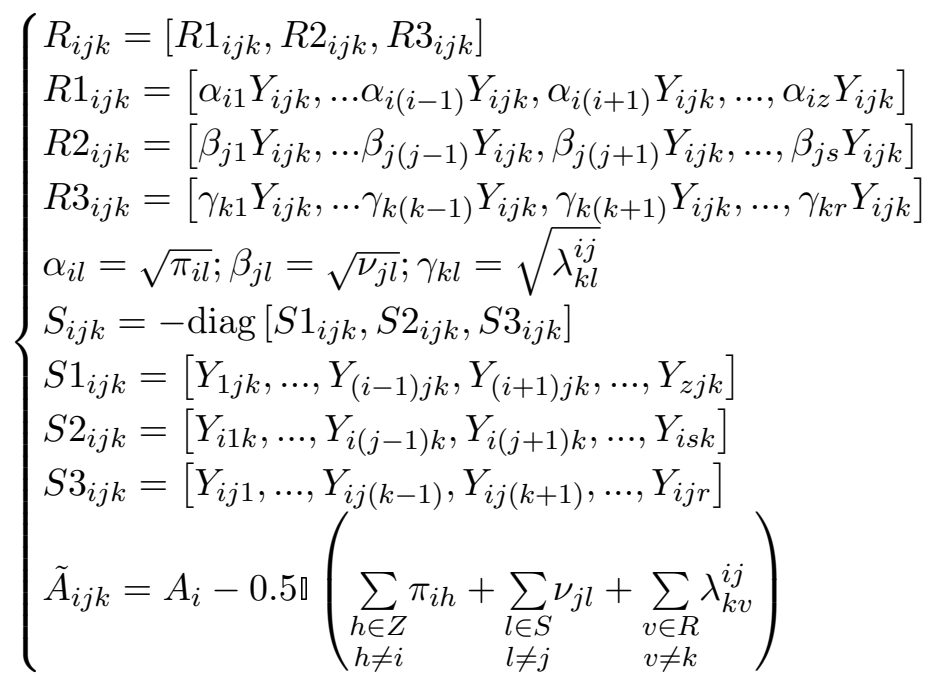

have feasible solutions $X_{i j k}=X_{i j k}^{T}, Y_{i j k}=Y_{i j k}^{T}, H_{i j k}$, and $F_{i j k}$. The corresponding compensator $\left(\varphi_{c}\right)$ is given by:

$$
\begin{gathered}
A_{c i j k}=\left(X_{i j k}-Y_{i j k}^{-1}\right)^{-1}\left[\tilde{A}_{i j k}^{T}+X_{i j k} \tilde{A}_{i j k} Y_{i j k}+X_{i j k} B_{j} F_{i j k}+H_{i j k} C_{2} Y_{i j k}+\left(\sum_{\substack{h \in Z \\
h \neq i}} \pi_{i h} Y_{h j k}^{-1}\right.\right. \\
\left.\left.+\sum_{\substack{l \in S \\
l \neq j}} \nu_{j l} Y_{i l k}^{-1}+\sum_{\substack{v \in R \\
v \neq k}} \lambda_{k v}^{i j} Y_{i j v}^{-1}\right) Y_{i j k}\right] Y_{i j k}^{-1}+0.5 I\left(\sum_{\substack{h \in Z \\
h \neq i}} \pi_{i h}+\sum_{\substack{l \in S \\
l \neq j}} \nu_{j l}+\sum_{\substack{v \in R \\
v \neq k}} \lambda_{k v}^{i j}\right) \\
B_{c i j k}=\left(Y_{i j k}^{-1}-X_{i j k}\right)^{-1} H_{i j k}, \quad C_{c i j k}=F_{i j k} Y_{i j k}^{-1}
\end{gathered}
$$

Proof see (Aberkane et al. 2005).

\subsection{Robust Internal Stochastic Stability}

In this part, we shall address the problem of characterising all dynamical compensators $\varphi_{c}$ that internally robustly exponentially stabilize the uncertain AFTCSMP in the mean square sense. The uncertainties we consider here are, respectively, structured ((NBU) and (LCU) uncertainties) and unstructured uncertainties ((VBU) uncertainties). The motivation to consider the above three different uncertainty forms is to provide a more unified treatment to handle both structured and unstructured parameter uncertainties.

Before giving a characterization of the dynamical compensators $\varphi_{c}$, we first introduce the following proposition which gives a necessary and sufficient condition for internal robust exponential stability in the mean square sense for the system (3), in term of coupled matrix inequalities. Indeed, the LMI characterization of the compensators is based on this condition.

Proposition 4.3 A necessary and sufficient condition for IRESS of the system (3) is that there exist symmetric positive-definite matrices $P_{i j k}$ and some positive constants $\gamma_{i}, i \in Z, j \in S$ and $k \in R$ such that 
a) (NBU) uncertainties

$$
\tilde{\Lambda}_{i j k}^{T} P_{i j k}+P_{i j k} \tilde{\Lambda}_{i j k}+\sum_{\substack{h \in Z \\ h \neq i}} \pi_{i h} P_{h j k}+\sum_{\substack{l \in S \\ l \neq j}} \nu_{j l} P_{i l k}+\sum_{\substack{v \in R \\ v \neq k}} \lambda_{k v}^{i j} P_{i j v}+\gamma_{i} P_{i j k} \bar{D}_{i} P_{i j k}+\left(1 / \gamma_{i}\right) \bar{K}_{i}<0
$$

b) (LCU) uncertainties

$$
\tilde{\Lambda}_{i j k}^{T} P_{i j k}+P_{i j k} \tilde{\Lambda}_{i j k}+\sum_{\substack{h \in Z \\ h \neq i}} \pi_{i h} P_{h j k}+\sum_{\substack{l \in S \\ l \neq j}} \nu_{j l} P_{i l k}+\sum_{\substack{v \in R \\ v \neq k}} \lambda_{k v}^{i j} P_{i j v}+\bar{a}_{i} \gamma_{i} P_{i j k} \sum_{l=1}^{p_{\Delta}} \bar{d}_{l i} \bar{d}_{l i}^{T} P_{i j k}+\left(\bar{a}_{i} / \gamma_{i}\right) \sum_{l=1}^{p_{\Delta}} \bar{v}_{l i} \bar{v}_{l i}^{T}<0
$$

c) (VBU) uncertainties

$$
\tilde{\Lambda}_{i j k}^{T} P_{i j k}+P_{i j k} \tilde{\Lambda}_{i j k}+\sum_{\substack{h \in Z \\ h \neq i}} \pi_{i h} P_{h j k}+\sum_{\substack{l \in S \\ l \neq j}} \nu_{j l} P_{i l k}+\sum_{\substack{v \in R \\ v \neq k}} \lambda_{k v}^{i j} P_{i j v}+\gamma_{i} P_{i j k} P_{i j k}+\left(n / \gamma_{i}\right) \operatorname{diag}\left(\bar{M}_{i}^{T} \bar{M}_{i}\right)<0
$$

Where: $\quad \bar{D}_{i}=\bar{H}_{i} \bar{H}_{i}^{T}, \bar{H}_{i}=\left[\begin{array}{c}H_{i} \\ \mathbf{0}\end{array}\right], \bar{K}_{i}=\bar{G}_{i}^{T} \bar{G}_{i}, \bar{G}_{i}=\left[G_{i} \mathbf{0}\right], \bar{d}_{l i}=\left[\begin{array}{c}d_{l i} \\ \mathbf{0}\end{array}\right], \bar{v}_{l i}=\left[\begin{array}{c}v_{l i} \\ \mathbf{0}\end{array}\right], \bar{M}_{i}=\left[M_{i} \mathbf{0}\right]$.

Proof We will only develop the proof for the case of (NBU) uncertainties. The same arguments can be used for the other forms of uncertainties.

Let a quadratic stochastic Lyapunov function:

$$
\vartheta\left(\chi_{t}, \xi_{t}, \eta_{t}, \psi_{t}\right)=\chi_{t}^{T} P\left(\xi_{t}, \eta_{t}, \psi_{t}\right) \chi_{t}
$$

where $P\left(\xi_{t}, \eta_{t}, \psi_{t}\right)$ is a positive symmetric matrix $\forall \xi_{t}=i \in Z, \eta_{t}=j \in R$ and $\psi_{t}=k \in R$. Evaluating $\mathcal{L} \vartheta\left(\chi_{t}, \xi_{t}, \eta_{t}, \psi_{t}\right)$ for the system (3), when the quantities $\xi_{t}=i \in Z, \eta_{t}=j \in R$ and $\psi_{t}=k \in R$ have occurred at some $t \in[0, \infty)$, we have:

$$
\mathcal{L} \vartheta=\chi_{t}^{T}\left\{\tilde{\Lambda}_{i j k}^{T} P_{i j k}+P_{i j k} \tilde{\Lambda}_{i j k}+\sum_{\substack{h \in Z \\ h \neq i}} \pi_{i h} P_{h j k}+\sum_{\substack{l \in S \\ l \neq j}} \nu_{j l} P_{i l k}+\sum_{\substack{v \in R \\ v \neq k}} \lambda_{k v}^{i j} P_{i j v}+2 P_{i j k} \Delta \Lambda_{i}\right\} \chi
$$

Note that, due to presence of the parameter uncertainties, the weak infinitesimal operator of the uncertain AFTCSMP contains an additional term in comparison with that of the certain AFTCSMP, that is

$$
\mathcal{L}_{u} \vartheta\left(\chi_{t}, \xi_{t}, \eta_{t}, \psi_{t}\right)=\mathcal{L}_{c} \vartheta\left(\chi_{t}, \xi_{t}, \eta_{t}, \psi_{t}\right)+\Delta \mathcal{L} \vartheta\left(\chi_{t}, \xi_{t}, \eta_{t}, \psi_{t}\right)
$$

where $\mathcal{L}_{u} \vartheta\left(\chi_{t}, \xi_{t}, \eta_{t}, \psi_{t}\right)$ is the weak infinitesimal operator of the uncertain AFTCSMP, $\mathcal{L}_{c} \vartheta\left(\chi_{t}, \xi_{t}, \eta_{t}, \psi_{t}\right)$ is the weak infinitesimal operator of the certain AFTCSMP and $\Delta \mathcal{L} \vartheta\left(\chi_{t}, \xi_{t}, \eta_{t}, \psi_{t}\right)$ is the part of the weak infinitesimal operator associated with the parameter uncertainties. This part has an upper bound, $(\Delta \mathcal{L} \vartheta)_{\text {bound }}$. Then

$$
\mathcal{L}_{u} \vartheta\left(\chi_{t}, \xi_{t}, \eta_{t}, \psi_{t}\right) \leq \mathcal{L}_{u p} \vartheta\left(\chi_{t}, \xi_{t}, \eta_{t}, \psi_{t}\right)=\mathcal{L}_{c} \vartheta\left(\chi_{t}, \xi_{t}, \eta_{t}, \psi_{t}\right)+(\Delta \mathcal{L} \vartheta)_{\text {bound }}
$$

where $\mathcal{L}_{\text {up }} \vartheta\left(\chi_{t}, \xi_{t}, \eta_{t}, \psi_{t}\right)$ is the upper bound of the weak infinitesimal operator with the maximum uncertainty values. From equation (14), the term due to the parameter uncertainties is

$$
\Delta \mathcal{L} \vartheta\left(\chi_{t}, i, j, k\right)=2 \chi_{t}^{T} P_{i j k} \Delta \Lambda_{i} \chi_{t}
$$

Using lemma 3.3, the upper bound of $\Delta \mathcal{L} \vartheta\left(\chi_{t}, i, j, k\right)$ is

$$
2 \chi_{t}^{T} P_{i j k} \Delta \Lambda_{i} \chi_{t}=2 \chi_{t}^{T} P_{i j k} \bar{H}_{i} F_{i} \bar{G}_{i} \chi_{t} \leq \chi_{t}^{T}\left[\gamma_{i} P_{i j k} \bar{H}_{i} \bar{H}_{i}^{T} P_{i j k}+\left(1 / \gamma_{i}\right) \bar{G}_{i}^{T} \bar{G}_{i}\right] \chi_{t}
$$


Therefore: $\left(\Delta \mathcal{L} \vartheta\left(\chi_{t}, i, j, k\right)\right)_{\text {bound }}=\chi_{t}^{T}\left\{\gamma_{i} P_{i j k} \bar{D}_{i} P_{i j k}+\left(1 / \gamma_{i}\right) \bar{K}_{i}\right\} \chi_{t}$.

Remark 2 For the other forms of uncertainties, $\left(\Delta \mathcal{L} \vartheta\left(\chi_{t}, i, j, k\right)\right)_{\text {bound }}$ is given as follows:

- (LCU) uncertainties: $\left(\Delta \mathcal{L} \vartheta\left(\chi_{t}, i, j, k\right)\right)_{b o u n d}=\chi_{t}^{T}\left\{\bar{a}_{i} \gamma_{i} P_{i j k} \sum_{l=1}^{p_{\Delta}} \bar{d}_{l i} \bar{d}_{l i}^{T} P_{i j k}+\left(\bar{a}_{i} / \gamma_{i}\right) \sum_{l=1}^{p_{\Delta}} \bar{v}_{l i} \bar{v}_{l i}^{T}\right\} \chi_{t}$.

- (VBU) uncertainties: $\left(\Delta \mathcal{L} \vartheta\left(\chi_{t}, i, j, k\right)\right)_{\text {bound }}=\chi_{t}^{T}\left\{\gamma_{i} P_{i j k} P_{i j k}+\left(n / \gamma_{i}\right) \operatorname{diag}\left(\bar{M}_{i}^{T} \bar{M}_{i}\right)\right\} \chi_{t}$.

a) Sufficiency

Assume that there exist $\gamma_{i}>0$ and $P_{i j k}>0, i \in Z, j \in S$ and $k \in R$ such that (10) is verified. Then $\vartheta\left(\chi_{t}, \xi_{t}, \eta_{t}, \psi_{t}\right)=\chi_{t}^{T} P\left(\xi_{t}, \eta_{t}, \psi_{t}\right) \chi_{t}$ is a stochastic Lyapunov function. From (10), we have

$$
\mathcal{L}_{\text {up }} \vartheta\left(\chi_{t}, \xi_{t}, \eta_{t}, \psi_{t}\right)=\mathcal{L}_{c} \vartheta\left(\chi_{t}, \xi_{t}, \eta_{t}, \psi_{t}\right)+(\Delta \mathcal{L} \vartheta)_{\text {bound }}<0
$$

then $\exists Q\left(\xi_{t}, \eta_{t}, \psi_{t}\right)>0$, such that $\mathcal{L}_{u p} \vartheta\left(\chi_{t}, \xi_{t}, \eta_{t}, \psi_{t}\right)=-\chi_{t}^{T} Q\left(\xi_{t}, \eta_{t}, \psi_{t}\right) \chi_{t}<0$. From (10), we obtain

$$
\mathcal{L}_{u} \vartheta\left(\chi_{t}, \xi_{t}, \eta_{t}, \psi_{t}\right) \leq-\chi_{t}^{T} Q\left(\xi_{t}, \eta_{t}, \psi_{t}\right) \chi_{t}
$$

For the quadratic Lyapunov function (13), we have

$$
\frac{\mathcal{L}_{u} \vartheta\left(\chi_{t}, \xi_{t}, \eta_{t}, \psi_{t}\right)}{\vartheta\left(\chi_{t}, \xi_{t}, \eta_{t}, \psi_{t}\right)} \leq \frac{-\chi_{t}^{T} Q\left(\xi_{t}, \eta_{t}, \psi_{t}\right) \chi_{t}}{\chi_{t}^{T} P\left(\xi_{t}, \eta_{t}, \psi_{t}\right) \chi_{t}}
$$

Taking the maximum, we obtain

$$
\frac{\mathcal{L}_{u} \vartheta\left(\chi_{t}, \xi_{t}, \eta_{t}, \psi_{t}\right)}{\vartheta\left(\chi_{t}, \xi_{t}, \eta_{t}, \psi_{t}\right)} \leq \max _{i, j, k}\left[\frac{-\chi_{t}^{T} Q\left(\xi_{t}, \eta_{t}, \psi_{t}\right) \chi_{t}}{\chi_{t}^{T} P\left(\xi_{t}, \eta_{t}, \psi_{t}\right) \chi_{t}}\right]=-\min _{i, j, k}\left[\frac{\lambda_{\min } Q\left(\xi_{t}, \eta_{t}, \psi_{t}\right)}{\lambda_{\max } P\left(\xi_{t}, \eta_{t}, \psi_{t}\right)}\right]=-\alpha<0
$$

where $\lambda_{\min } Q\left(\xi_{t}, \eta_{t}, \psi_{t}\right)$ is the eigenvalue of the matrix $Q\left(\xi_{t}, \eta_{t}, \psi_{t}\right)$ with the smallest real part, and $\lambda_{\max } P\left(\xi_{t}, \eta_{t}, \psi_{t}\right)$ is the eigenvalue of $P\left(\xi_{t}, \eta_{t}, \psi_{t}\right)$ with the largest real part, for all $i \in Z, j \in S$ and $k \in R . \alpha$ is a positive constant and represents the rate of convergence. We can rewrite (16) as

$$
\mathcal{L}_{u} \vartheta\left(\chi_{t}, \xi_{t}, \eta_{t}, \psi_{t}\right) \leq-\alpha \vartheta\left(\chi_{t}, \xi_{t}, \eta_{t}, \psi_{t}\right)
$$

From Dynkin's formula and the Gronwell-Bellman lemma (Mahmoud et al. 2003), we have

$$
\mathcal{E}\left\{\vartheta\left(\chi_{t}, \xi_{t}, \eta_{t}, \psi_{t}\right)\right\} \leq \exp \left(-\epsilon\left(t-t_{0}\right)\right) \vartheta\left(\chi_{0}, \xi_{0}, \eta_{0}, \psi_{0}, t\right)
$$

or

$$
\mathcal{E}\left\{\left\|\chi\left(t ; \chi_{0}, t_{0} \|^{2}\right\} \leq b\right\| \chi_{0} \|^{2} \exp \left[-\epsilon\left(t-t_{0}\right)\right]\right.
$$

where $b$ and $\epsilon$ are positive constants.

Then, by definition 3.2, the dynamical system (3) is IRESS $\forall t>t_{0}$.

b) Necessity

Assume that (3) is internally robustly exponentially stable in the mean square sense, then using same arguments as in (Srichander et al. 1993) (Lemma 4.2), for any given positive-definite matrices $Q_{i j k}, i \in Z$, $j \in S$ and $k \in R$, there exist symmetric positive-definite matrices $P_{i j k}, i \in Z, j \in S$ and $k \in R$ such that

$$
\mathcal{L}_{u p} \vartheta\left(\chi_{t}, \xi_{t}, \eta_{t}, \psi_{t}\right)=-\chi_{t}^{T} Q_{i j k} \chi_{t}<0
$$

where $\vartheta\left(\chi_{t}, \xi_{t}, \eta_{t}, \psi_{t}\right)$ is given by (13). From (17), we conclude that there exist symmetric positive-definite matrices $P_{i j k}, i \in Z, j \in S$ and $k \in R$ and some positive constants $\gamma_{i}, \forall i \in Z$ such that (10) is verified. 
Hence the proof is complete.

The following proposition gives an LMI characterization of dynamical compensators $\left(\varphi_{c}\right)$ that internally robustly stabilize the closed-loop system in the mean square sense.

Proposition 4.4 A necessary and sufficient condition for internal robust exponential stability in the mean square sense of the system (3) is that the following matrix inequalities

$$
\left[\begin{array}{ccc}
\phi_{i j k} & \Delta_{i j k}^{T} & R_{i j k}(Y) \\
\star & -\gamma_{i} \rrbracket & \mathbf{0} \\
\star & \star & S_{i j k}(Y)
\end{array}\right]<0, \quad\left[\begin{array}{cc}
\theta_{i j k} & \Xi_{i j k} \\
\star & -\left(1 / \gamma_{i}\right) \rrbracket
\end{array}\right]<0, \quad\left[\begin{array}{cc}
Y_{i j k} & \mathbb{} \\
\star & X_{i j k}
\end{array}\right]>0
$$

where

a) (NBU) uncertainties

$$
\left\{\begin{aligned}
\phi_{i j k} & =\tilde{A}_{i j k} Y_{i j k}+Y_{i j k} \tilde{A}_{i j k}^{T}+F_{i j k}^{T} B_{j}^{T}+B_{j} F_{i j k}+\gamma_{i} H_{i} H_{i}^{T} \\
\theta_{i j k} & =\tilde{A}_{i j k}^{T} X_{i j k}+X_{i j k} \tilde{A}_{i j k}+C_{2}^{T} L_{i j k}^{T}+L_{i j k} C_{2} \\
& +\left(1 / \gamma_{i}\right) G_{i}^{T} G_{i}+\sum_{\substack{h \in Z \\
h \neq i}} \pi_{i h} X_{h j k}+\sum_{\substack{l \in S \\
l \neq j}} \nu_{j l} X_{i l k}+\sum_{\substack{v \in R \\
v \neq k}} \lambda_{k v}^{i j} X_{i j v} \\
\Delta_{i j k} & =G_{i} Y_{i j k}, \quad \Xi_{i j k}=X_{i j k} H_{i}
\end{aligned}\right.
$$

b) (LCU) uncertainties

$$
\left\{\begin{array}{l}
\phi_{i j k}=\tilde{A}_{i j k} Y_{i j k}+Y_{i j k} \tilde{A}_{i j k}^{T}+F_{i j k}^{T} B_{j}^{T}+B_{j} F_{i j k}+\bar{a}_{i} \gamma_{i} W_{i} W_{i}^{T} \\
\theta_{i j k}=\tilde{A}_{i j k}^{T} X_{i j k}+X_{i j k} \tilde{A}_{i j k}+C_{2}^{T} L_{i j k}^{T}+L_{i j k} C_{2} \\
\quad+\left(\bar{a}_{i} / \gamma_{i}\right) T_{i}^{T} T_{i}+\sum_{\substack{h \in Z \\
h \neq i}} \pi_{i h} X_{h j k}+\sum_{\substack{l \in S \\
l \neq j}} \nu_{j l} X_{i l k}+\sum_{\substack{v \in R \\
v \neq k}} \lambda_{k v}^{i j} X_{i j v} \\
\\
W_{i}=\left[d_{1 i} \ldots d_{p_{\triangle i}}\right], \quad T_{i}=\left[v_{1 i} \vdots v_{p_{\triangle i}}\right]^{T}, \quad \Delta_{i j k}=\sqrt{\bar{a}_{i}} T_{i} Y_{i j k} \\
\Xi_{i j k}=\sqrt{\bar{a}_{i}} X_{i j k} W_{i}
\end{array}\right.
$$

c) (VBU) uncertainties

$$
\left\{\begin{aligned}
\phi_{i j k} & =\tilde{A}_{i j k} Y_{i j k}+Y_{i j k} \tilde{A}_{i j k}^{T}+F_{i j k}^{T} B_{j}^{T}+B_{j} F_{i j k}+\gamma_{i} I \\
\theta_{i j k} & =\tilde{A}_{i j k}^{T} X_{i j k}+X_{i j k} \tilde{A}_{i j k}+C_{2}^{T} L_{i j k}^{T}+L_{i j k} C_{2} \\
& +\left(n / \gamma_{i}\right) \operatorname{diag}\left(M_{i}^{T} M_{i}\right)+\sum_{\substack{h \in Z \\
h \neq i}} \pi_{i h} X_{h j k}+\sum_{\substack{l \in S \\
l \neq j}} \nu_{j l} X_{i l k}+\sum_{\substack{v \in R \\
l \in k \\
v \neq k}} \lambda_{k v}^{i j} X_{i j v} \\
\Delta_{i j k} & =\sqrt{n \operatorname{diag}\left(M_{i}^{T} M_{i}\right)} Y_{i j k}, \quad \Xi_{i j k}=X_{i j k}
\end{aligned}\right.
$$

have feasible solutions $X_{i j k}=X_{i j k}^{T}, Y_{i j k}=Y_{i j k}^{T}, L_{i j k}, F_{i j k}$ and $\gamma_{i}>0, \forall i \in Z, j \in S$ and $k \in R$. The corresponding compensator $\left(\varphi_{c}\right)$ is given by

$$
B_{c i j k}=\left(Y_{i j k}^{-1}-X_{i j k}\right)^{-1} L_{i j k}, \quad C_{c i j k}=F_{i j k} Y_{i j k}^{-1}
$$




$$
\begin{aligned}
& A_{c i j k}=\left(X_{i j k}-Y_{i j k}^{-1}\right)^{-1}\left[\tilde{A}_{i j k}^{T}+X_{i j k} \tilde{A}_{i j k} Y_{i j k}+X_{i j k} B_{j} F_{i j k}+L_{i j k} C_{2} Y_{i j k}+\left(\sum_{h \in Z} \pi_{i h} Y_{h j k}^{-1}\right.\right. \\
& h \neq i \\
& \left.\left.+\sum_{\substack{l \in S \\
l \neq j}} \nu_{j l} Y_{i l k}^{-1}+\sum_{\substack{v \in R \\
v \neq k}} \lambda_{k v}^{i j} Y_{i j v}^{-1}+\Sigma_{i j k}\right) Y_{i j k}+\Phi_{i j k}\right] Y_{i j k}^{-1}+0.5 \square\left(\sum_{\substack{h \in Z \\
h \neq i}} \pi_{i h}+\sum_{\substack{l \in S \\
l \neq j}} \nu_{j l}+\sum_{\substack{v \in R \\
v \neq k}} \lambda_{k v}^{i j}\right)
\end{aligned}
$$

where

- $(\mathrm{NBU})$ uncertainties: $\Sigma_{i j k}=\left(1 / \gamma_{i}\right) G_{i}^{T} G_{i}, \quad \Phi_{i j k}=\gamma_{i} X_{i j k} H_{i} H_{i}^{T}$.

- (LCU) uncertainties: $\Sigma_{i j k}=\left(\bar{a}_{i} / \gamma_{i}\right) T_{i}^{T} T_{i}, \quad \Phi_{i j k}=\bar{a}_{i} \gamma_{i} X_{i j k} W_{i} W_{i}^{T}$.

- (VBU) uncertainties: $\Sigma_{i j k}=\left(n / \gamma_{i}\right) \operatorname{diag}\left(M_{i}^{T} M_{i}\right), \quad \Phi_{i j k}=\gamma_{i} X_{i j k}$.

Proof The proof of this proposition follows the same arguments as for the proof of proposition 4.2.

\section{$5 \quad \mathcal{H}_{\infty}$ Control}

In this section, we deal with the design of controllers that stochastically stabilize the closed-loop system and guarantee the disturbance rejection, with a certain level $\mu>0$. This problematic is addressed under a convex optimization framework. Mathematically, we are concerned with the LMI characterization of dynamical compensators $\varphi_{c}$ that stochastically stabilize the system (3) and guarantee the following for all $w \in L^{2}[0, \infty):$

$$
\left\|z_{\infty}\right\|_{\mathcal{E}_{2}}=\mathcal{E}\left\{\int_{0}^{\infty} z_{\infty t}^{T} z_{\infty t} d t\right\}^{1 / 2}<\mu\left[\|w\|_{2}^{2}+a\left(\chi_{0}, \xi_{0}, \eta_{0}, \psi_{0}\right)\right]^{1 / 2}
$$

where $\mu>0$ is a prescribed level of disturbance attenuation to be achieved and $a\left(\chi_{0}, \xi_{0}, \eta_{0}, \psi_{0}\right)$ is a constant that depends on the initial conditions $\left(\chi_{0}, \xi_{0}, \eta_{0}, \psi_{0}\right)$. To this end, we need the auxiliary result given by the following proposition.

Proposition 5.1 If the system (3) is IESS, for every $w=\left\{w_{t} ; t \geq 0\right\} \in L_{2}[0, \infty)$, we have that $\chi=$ $\left\{\chi_{t} ; t \geq 0\right\} \in L_{2}((\Omega, \mathcal{F}, P),[0, \infty))$, i.e., $\mathcal{E}\left\{\int_{0}^{\infty} \chi_{t}^{T} \chi_{t} d t\right\}<\infty$, for any initial conditions $\left(\chi_{0}, \xi_{0}, \eta_{0}, \psi_{0}\right)$

Proof Since the system (3) is internally exponentially stable in the mean square sense, it follows from proposition 4.1 that there exist symmetric positive-definite matrices $P_{i j k}, i \in Z, j \in S$ and $k \in R$ such that (5) is verified.

Note that it is easy to show that there exists $\alpha>0$, such that

$$
\Xi_{i j k}+\alpha P_{i j k}^{2}<0
$$

$\forall i \in Z, j \in S$ and $k \in R$.

Let us consider the same quadratic stochastic Lyapunov function as in (13). Then

$$
\mathcal{L} \vartheta\left(\chi_{t}, \xi_{t}, \eta_{t}, \psi_{t}\right)=\chi_{t}^{T}\left\{\Xi\left(\xi_{t}, \eta_{t}, \psi_{t}\right)\right\} \chi_{t}+2 \chi_{t}^{T} P\left(\xi_{t}, \eta_{t}, \psi_{t}\right) \bar{E}\left(\xi_{t}, \eta_{t}, \psi_{t}\right) w_{t}
$$

Using lemma 3.3, it follows from (22) that

$$
\mathcal{L} \vartheta\left(\chi_{t}, \xi_{t}, \eta_{t}, \psi_{t}\right) \leq-\chi_{t}^{T} \Gamma\left(\xi_{t}, \eta_{t}, \psi_{t}\right) \chi_{t}+\alpha^{-1} w_{t}^{T} \bar{E}\left(\xi_{t}, \eta_{t}, \psi_{t}\right)^{T} \bar{E}\left(\xi_{t}, \eta_{t}, \psi_{t}\right) w_{t}
$$


where $\Gamma\left(\xi_{t}, \eta_{t}, \psi_{t}\right)=-\Xi\left(\xi_{t}, \eta_{t}, \psi_{t}\right)-\alpha P^{2}\left(\xi_{t}, \eta_{t}, \psi_{t}\right)$. From Dynkin's formula, we have

$$
\begin{aligned}
\mathcal{E}\left\{\vartheta\left(\chi_{T}, \xi_{T}, \eta_{T}, \psi_{T}\right)\right\}-\vartheta\left(\chi_{0}, \xi_{0}, \eta_{0}, \psi_{0}\right) & =\mathcal{E}\left\{\int_{0}^{T} \mathcal{L} \vartheta\left(\chi_{\tau}, \xi_{\tau}, \eta_{\tau}, \psi_{\tau}\right) d \tau\right\} \\
& \leq-\mathcal{E}\left\{\int_{0}^{T} \chi_{\tau}^{T} \Gamma\left(\xi_{\tau}, \eta_{\tau}, \psi_{\tau}\right) \chi_{\tau} d \tau\right\} \\
& +\alpha^{-1} \mathcal{E}\left\{\int_{0}^{T} w_{\tau}^{T} \bar{E}\left(\xi_{\tau}, \eta_{\tau}, \psi_{\tau}\right)^{T} \bar{E}\left(\xi_{\tau}, \eta_{\tau}, \psi_{\tau}\right) w_{\tau} d \tau\right\} \\
& \leq-\mathcal{E}\left\{\int_{0}^{T} \lambda_{\min } \Gamma\left(\xi_{\tau}, \eta_{\tau}, \psi_{\tau}\right) \chi_{\tau}^{T} \chi_{\tau} d \tau\right\} \\
& +\alpha^{-1} \mathcal{E}\left\{\int_{0}^{T} \lambda_{\max }\left(\bar{E}\left(\xi_{\tau}, \eta_{\tau}, \psi_{\tau}\right)^{T} \bar{E}\left(\xi_{\tau}, \eta_{\tau}, \psi_{\tau}\right)\right) w_{\tau}^{T} w_{\tau} d \tau\right\}
\end{aligned}
$$

From (23), we get

$$
\begin{aligned}
& \lim _{T \rightarrow \infty}\left\{\mathcal{E}\left\{\chi_{T}^{T} P\left(\xi_{T}, \eta_{T}, \psi_{T}\right) \chi_{T}\right\}+\min _{i, j, k}\left\{\lambda_{\min } \Gamma(i, j, k)\right\} \mathcal{E}\left\{\int_{0}^{T} \chi_{\tau}^{T} \chi_{\tau} d \tau\right\}\right\} \\
& \leq\left\{\chi_{0}^{T} P\left(\xi_{0}, \eta_{0}, \psi_{0}\right) \chi_{0}\right\}+\alpha^{-1} \max _{i, j, k}\left\{\lambda_{\max } \bar{E}^{T}(i, j, k) \bar{E}(i, j, k)\right\} \mathcal{E}\left\{\int_{0}^{\infty} w_{\tau}^{T} w_{\tau} d \tau\right\}
\end{aligned}
$$

From (24), and knowing that $\mathcal{E}\left\{\chi_{t}^{T} P\left(\xi_{t}, \eta_{t}, \psi_{t}\right) \chi_{t}\right\} \geq 0$ and $w(\cdot) \in L^{2}[0, \infty)$, then the system (3) is stochastically stable. Hence the proof is complete.

Proposition 5.2 If there exist symmetric positive-definite matrices $P_{i j k}, i \in Z, j \in S$ and $k \in R$ such that

$$
\tilde{\Lambda}_{i j k}^{T} P_{i j k}+P_{i j k} \tilde{\Lambda}_{i j k}+\bar{C}_{1 j k}^{T} \bar{C}_{1 j k}+\mu^{-2} P_{i j k} \bar{E}_{i j k} \bar{E}_{i j k}^{T} P_{i j k}+\sum_{\substack{h \in Z \\ h \neq i}} \pi_{i h} P_{h j k}+\sum_{\substack{l \in S \\ l \neq j}} \nu_{j l} P_{i l k}+\sum_{\substack{v \in R \\ v \neq k}} \lambda_{k v}^{i j} P_{i j v}=\Pi_{i j k}<0
$$

then the system (3) (when the uncertainties are equal to zero) is stochastically stable and satisfies

$$
\left\|z_{\infty}\right\|_{\mathcal{E}_{2}}<\left[\mu^{2}\|w\|_{2}^{2}+\chi_{0}^{T} P\left(\xi_{0}, \eta_{0}, \psi_{0}\right) \chi_{0}\right]^{1 / 2}
$$

Proof Since $\bar{C}_{1 j k}^{T} \bar{C}_{1 j k}+\mu^{-2} P_{i j k} \bar{E}_{i j k} \bar{E}_{i j k}^{T} P_{i j k} \geq 0$, it follows from (25) and proposition 4.1, that the system (3) is internally exponentially stable in the mean square sense. Using proposition 5.1, it follows that (3) is stochastically stable.

Let us now prove that (26) is verified. We begin by defining the following function

$$
\mathcal{J}_{T}=\mathcal{E}\left\{\int_{0}^{T}\left(z_{\infty t}^{T} z_{\infty t}-\mu^{2} w_{t}^{T} w_{t}\right) d t\right\}
$$

Then, to prove (26), it suffices to establish that: $\mathcal{J}_{\infty} \leq \chi_{0}^{T} P\left(\xi_{0}, \eta_{0}, \psi_{0}\right) \chi_{0}$.

Let a quadratic stochastic Lyapunov function as defined in (13), then:

$$
\mathcal{L} \vartheta\left(\chi_{t}, \xi_{t}, \eta_{t}, \psi_{t}\right)=\chi_{t}^{T}\left\{\Xi\left(\xi_{t}, \eta_{t}, \psi_{t}\right)\right\} \chi_{t}+2 \chi_{t}^{T} P\left(\xi_{t}, \eta_{t}, \psi_{t}\right) \bar{E}\left(\xi_{t}, \eta_{t}, \psi_{t}\right) w_{t}
$$


adding and subtracting $\mathcal{E}\left\{\int_{0}^{T} \mathcal{L} \vartheta\left(\chi_{t}, \xi_{t}, \eta_{t}, \psi_{t}\right) d t\right\}$ to $(27)$, we get

$$
\begin{aligned}
\mathcal{J}_{T} & =\mathcal{E}\left\{\int_{0}^{T} \chi_{t}^{T}\left[\Xi\left(\xi_{t}, \eta_{t}, \psi_{t}\right)+\bar{C}_{1}\left(\eta_{t}, \psi_{t}\right)^{T} \bar{C}_{1}\left(\eta_{t}, \psi_{t}\right)\right] \chi_{t}\right. \\
& +\mu^{-2} \chi_{t}^{T} P\left(\xi_{t}, \eta_{t}, \psi_{t}\right) \bar{E}\left(\xi_{t}, \eta_{t}, \psi_{t}\right) \bar{E}\left(\xi_{t}, \eta_{t}, \psi_{t}\right)^{T} P\left(\xi_{t}, \eta_{t}, \psi_{t}\right) \chi_{t} \\
& -\mu^{2}\left(w_{t}-\mu^{-2} \bar{E}\left(\xi_{t}, \eta_{t}, \psi_{t}\right)^{T} P\left(\xi_{t}, \eta_{t}, \psi_{t}\right) \chi_{t}\right)^{T} \\
& \left.\left(w_{t}-\mu^{-2} \bar{E}\left(\xi_{t}, \eta_{t}, \psi_{t}\right)^{T} P\left(\xi_{t}, \eta_{t}, \psi_{t}\right) \chi_{t}\right) d t\right\}-\mathcal{E}\left\{\int_{0}^{T} \mathcal{L} \vartheta\left(\chi_{t}, \xi_{t}, \eta_{t}, \psi_{t}\right) d t\right\} \\
& =\mathcal{E}\left\{\int_{0}^{T} \chi_{t}^{T}\left[\Pi\left(\xi_{t}, \eta_{t}, \psi_{t}\right)\right] \chi_{t}-\mu^{2}\left(w_{t}-\mu^{-2} \bar{E}\left(\xi_{t}, \eta_{t}, \psi_{t}\right)^{T} P\left(\xi_{t}, \eta_{t}, \psi_{t}\right) \chi_{t}\right)^{T}\right. \\
& \left.\left(w_{t}-\mu^{-2} \bar{E}\left(\xi_{t}, \eta_{t}, \psi_{t}\right)^{T} P\left(\xi_{t}, \eta_{t}, \psi_{t}\right) \chi_{t}\right) d t\right\}-\mathcal{E}\left\{\int_{0}^{T} \mathcal{L} \vartheta\left(\chi_{t}, \xi_{t}, \eta_{t}, \psi_{t}\right) d t\right\}
\end{aligned}
$$

From Dynkin's formula, we have

$$
\mathcal{E}\left\{\vartheta\left(\chi_{T}, \xi_{T}, \eta_{T}, \psi_{T}\right)\right\}-\vartheta\left(\chi_{0}, \xi_{0}, \eta_{0}, \psi_{0}\right)=\mathcal{E}\left\{\int_{0}^{T} \mathcal{L} \vartheta\left(\xi_{t}, \eta_{t}, \psi_{t}\right) d t\right\}
$$

Since $\Pi\left(\xi_{t}, \eta_{t}, \psi_{t}\right)<0$ and $\mathcal{E}\left\{\vartheta\left(\chi_{T}, \xi_{T}, \eta_{T}, \psi_{T}\right)\right\} \geq 0$, it follows from (29) and (30) that

$$
\mathcal{J}_{T} \leq \vartheta\left(\chi_{0}, \xi_{0}, \eta_{0}, \psi_{0}\right)
$$

which yields $\mathcal{J}_{\infty} \leq \chi_{0}^{T} P\left(\xi_{0}, \eta_{0}, \psi_{0}\right) \chi_{0}$. Hence the proof is complete.

The $\mathcal{H}_{\infty}$ constraints (26) can be rephrased in LMI form. This is illustrated by proposition 5.3, which gives an LMI characterization of output feedback dynamical compensators $\left(\varphi_{c}\right)$ that stochastically stabilize the AFTCSMP and ensures (26).

Proposition 5.3 The $\mathcal{H}_{\infty}$ constraints (25) are equivalent to that (31)-(32)

$$
\begin{gathered}
{\left[\begin{array}{ccc}
\bar{\phi}_{i j k} & \left(C_{1} Y_{i j k}+D_{1 j} F_{i j k}\right)^{T} & R_{i j k}(Y) \\
\left(C_{1} Y_{i j k}+D_{1 j} F_{i j k}\right) & -I & 0 \\
R_{i j k}(Y)^{T} & 0 & S_{i j k}(Y)
\end{array}\right]<0} \\
{\left[\begin{array}{cc}
\bar{\theta}_{i j k} & \left(X_{i j k} E_{i j}+L_{i j k} D_{2 i j}\right) \\
\left(X_{i j k} E_{i j}+L_{i j k} D_{2 i j}\right)^{T} & -\mu^{2} I
\end{array}\right]<0,} \\
{\left[\begin{array}{cc}
Y_{i j k} & I \\
I & X_{i j k}
\end{array}\right]>0}
\end{gathered}
$$

where

$$
\left\{\begin{array}{c}
\bar{\phi}_{i j k}=\tilde{A}_{i j k} Y_{i j k}+Y_{i j k} \tilde{A}_{i j k}^{T}+F_{i j k}^{T} B_{j}^{T}+B_{j} F_{i j k}+\mu^{-2} E_{i j} E_{i j}^{T} \\
\bar{\theta}_{i j k}=\tilde{A}_{i j k}^{T} X_{i j k}+X_{i j k} \tilde{A}_{i j k}+C_{2}^{T} L_{i j k}^{T}+L_{i j k} C_{2} \\
\quad+C_{1}^{T} C_{1}+\sum_{\substack{h \in Z \\
h \neq i}} \pi_{i h} X_{h j k}+\sum_{\substack{l \in S \\
l \neq j}} \nu_{j l} X_{i l k}+\sum_{\substack{v \in R \\
v \neq k}} \lambda_{k v}^{i j} X_{i j v}
\end{array}\right.
$$

have feasible solutions $X_{i j k}=X_{i j k}^{T}, Y_{i j k}=Y_{i j k}^{T}, L_{i j k}$ and $F_{i j k}, \forall i \in Z, j \in S$ and $k \in R$. The corresponding compensator $\left(\varphi_{c}\right)$ is given by

$$
B_{c i j k}=\left(Y_{i j k}^{-1}-X_{i j k}\right)^{-1} L_{i j k}, \quad C_{c i j k}=F_{i j k} Y_{i j k}^{-1}
$$




$$
\begin{aligned}
& A_{c i j k}=\left(X_{i j k}-Y_{i j k}^{-1}\right)^{-1}\left[\tilde{A}_{i j k}^{T}+X_{i j k} \tilde{A}_{i j k} Y_{i j k}+X_{i j k} B_{j} F_{i j k}+L_{i j k} C_{2} Y_{i j k}+\left(\sum_{h \in Z} \pi_{i h} Y_{h j k}^{-1}\right.\right. \\
& \\
& h \neq i \\
&+\left.\left.\sum_{\substack{l \in S \\
l \neq j}} \nu_{j l} Y_{i l k}^{-1}+\sum_{\substack{v \in R \\
v \neq k}} \lambda_{k v}^{i j} Y_{i j v}^{-1}\right) Y_{i j k}+C_{1}^{T}\left(C_{1} Y_{i j k}+D_{1 j} F_{i j k}\right)+\mu^{-2}\left(X_{i j k} E_{i j}+L_{i j k} D_{2 i j}\right)\right] Y_{i j k}^{-1} \\
&+0.5 I\left(\sum_{\substack{h \in Z \\
h \neq i}} \pi_{i h}+\sum_{\substack{l \in S \\
l \neq j}} \nu_{j l}+\sum_{\substack{v \in R \\
v \neq k}} \lambda_{k v}^{i j}\right)
\end{aligned}
$$

Proof The proof of this proposition follows essentially the same lines as for the proof of proposition 4.2. $\square$

\section{Robust $\mathcal{H}_{\infty}$ Control}

In this section, we investigate the robust $\mathcal{H}_{\infty}$ control of uncertain AFTCSMP subject to both structured ((NBU) and (LCU) uncertainties) and unstructured uncertainties ((VBU) uncertainties). It is shown that the above problematic can be recast as a convex optimization problem characterized by LMI, providing thus a characterization of output feedback dynamical compensators that robustly stochastically stabilize the AFTCSMP and ensures (26) for all admissible uncertainties.

The robust $\mathcal{H}_{\infty}$ control problem addressed in this paper can be formulated as follows: given a prescribed level of disturbance attenuation $\mu>0$, construct a dynamic output feedback controller of the form (2) such that the following two requirements are satisfied:

a) The uncertain AFTCSMP (3) is robustly stochastically stable;

b) (26) is satisfied for all $w \in L^{2}[0, \infty)$ and all admissible uncertainties.

Before introducing the sufficient condition for the solvability of the robust $\mathcal{H}_{\infty}$ control problem, we first give the following proposition which will play a key role in the derivation of our main results.

Proposition 6.1 If the system (3) is IRESS, for every $w=\left\{w_{t} ; t \geq 0\right\} \in L_{2}[0, \infty)$, we have that $\chi=$ $\left\{\chi_{t} ; t \geq 0\right\} \in L_{2}((\Omega, \mathcal{F}, P),[0, \infty))$, i.e., $\mathcal{E}\left\{\int_{0}^{\infty} \chi_{t}^{T} \chi_{t} d t\right\}<\infty$, for any initial conditions $\left(\chi_{0}, \xi_{0}, \eta_{0}, \psi_{0}\right)$ and for all admissible uncertainties.

Proof The proof of this proposition follows the same arguments as for the proof of proposition 5.1.

PROPOSITION 6.2 If there exist symmetric positive-definite matrices $P_{i j k}$ and some positive constants $\gamma_{i}$, $i \in Z, j \in S$ and $k \in R$ such that

a) (NBU) uncertainties

$$
\begin{aligned}
\tilde{\Lambda}_{i j k}^{T} P_{i j k}+P_{i j k} \tilde{\Lambda}_{i j k} & +\bar{C}_{1 j k}^{T} \bar{C}_{1 j k}+\mu^{-2} P_{i j k} \bar{E}_{i j k} \bar{E}_{i j k}^{T} P_{i j k}+\gamma_{i} P_{i j k} \bar{D}_{i} P_{i j k} \\
& +\gamma_{i}^{-1} \bar{K}_{i}+\sum_{\substack{h \in Z \\
h \neq i}} \pi_{i h} P_{h j k}+\sum_{\substack{l \in S \\
l \neq j}} \nu_{j l} P_{i l k}+\sum_{\substack{v \in R \\
v \neq k \\
v \neq k}} \lambda_{k v}^{i j} P_{i j v}<0
\end{aligned}
$$


b) (LCU) uncertainties

$$
\begin{aligned}
\tilde{\Lambda}_{i j k}^{T} P_{i j k}+P_{i j k} \tilde{\Lambda}_{i j k} & +\bar{C}_{1 j k}^{T} \bar{C}_{1 j k}+\mu^{-2} P_{i j k} \bar{E}_{i j k} \bar{E}_{i j k}^{T} P_{i j k}+\bar{a}_{i} \gamma_{i} P_{i j k} \sum_{l=1}^{p_{\Delta}} \bar{d}_{l i} \bar{d}_{l i}^{T} P_{i j k} \\
& +\left(\bar{a}_{i} / \gamma_{i}\right) \sum_{l=1}^{p_{\Delta}} \bar{v}_{l i} \bar{v}_{l i}^{T}+\sum_{\substack{h \in Z \\
h \neq i}} \pi_{i h} P_{h j k}+\sum_{\substack{l \in S \\
l \neq j}} \nu_{j l} P_{i l k}+\sum_{\substack{v \in R \\
v \neq k}} \lambda_{k v}^{i j} P_{i j v}<0
\end{aligned}
$$

c) (VBU) uncertainties

$$
\begin{aligned}
\tilde{\Lambda}_{i j k}^{T} P_{i j k}+P_{i j k} \tilde{\Lambda}_{i j k} & +\bar{C}_{1 j k}^{T} \bar{C}_{1 j k}+\mu^{-2} P_{i j k} \bar{E}_{i j k} \bar{E}_{i j k}^{T} P_{i j k}+\gamma_{i} P_{i j k} P_{i j k} \\
& +\left(n / \gamma_{i}\right) \operatorname{diag}\left(\bar{M}_{i}^{T} \bar{M}_{i}\right)+\sum_{\substack{h \in Z \\
h \neq i}} \pi_{i h} P_{h j k}+\sum_{\substack{l \in S \\
l \neq j}} \nu_{j l} P_{i l k}+\sum_{\substack{v \in R \\
v \neq k}} \lambda_{k v}^{i j} P_{i j v}<0
\end{aligned}
$$

$\forall i \in Z, j \in S$ and $k \in R$.

then the system (3) is robustly stochastically stable and satisfies

$$
\left\|z_{\infty}\right\|_{\mathcal{E}_{2}}<\left[\mu^{2}\|w\|_{2}^{2}+\chi_{0}^{T} P\left(\xi_{0}, \eta_{0}, \psi_{0}\right) \chi_{0}\right]^{1 / 2}
$$

for all admissible uncertainties.

Proof The proof of this proposition follows the same lines as for the proof of proposition 5.2.

Proposition 6.3 The constraints (35) ((36) and (37), respectively) are equivalent to the following matrix inequalities

$$
\begin{aligned}
& {\left[\begin{array}{cccc}
\Upsilon_{i j k} & \left(C_{1} Y_{i j k}+D_{1 j} F_{i j k}\right)^{T} & \Delta_{i j k}^{T} & R_{i j k}(Y) \\
\star & -\rrbracket & \mathbf{0} & \mathbf{0} \\
\star & \star & -\gamma_{i} \rrbracket & \mathbf{0} \\
\star & \star & \star & S_{i j k}(Y)
\end{array}\right]<0, \quad\left[\begin{array}{ccc}
\Omega_{i j k} & \left(X_{i j k} E_{i j}+L_{i j k} D_{2 i j}\right) & \Xi_{i j k} \\
\star & -\mu^{2} \rrbracket & \mathbf{0} \\
\star & \star & -\left(1 / \gamma_{i}\right) \square
\end{array}\right]<0} \\
& {\left[\begin{array}{cc}
Y_{i j k} & \mathbb{} \\
\star & X_{i j k}
\end{array}\right]>0}
\end{aligned}
$$

where

a) (NBU) uncertainties

$$
\left\{\begin{array}{c}
\Upsilon_{i j k}=\tilde{A}_{i j k} Y_{i j k}+Y_{i j k} \tilde{A}_{i j k}^{T}+F_{i j k}^{T} B_{j}^{T}+B_{j} F_{i j k}+\mu^{-2} E_{i j} E_{i j}^{T}+\gamma_{i} H_{i} H_{i}^{T} \\
\Omega_{i j k}=\tilde{A}_{i j k}^{T} X_{i j k}+X_{i j k} \tilde{A}_{i j k}+C_{2}^{T} L_{i j k}^{T}+L_{i j k} C_{2} \\
\quad+C_{1}^{T} C_{1}+\left(1 / \gamma_{i}\right) G_{i}^{T} G_{i}+\sum_{\substack{h \in Z \\
h \neq i}} \pi_{i h} X_{h j k}+\sum_{\substack{l \in S \\
l \neq j}} \nu_{j l} X_{i l k}+\sum_{\substack{v \in R \\
v \neq k \\
v \neq k}} \lambda_{k v}^{i j} X_{i j v}
\end{array}\right.
$$

b) (LCU) uncertainties

$$
\left\{\begin{array}{c}
\Upsilon_{i j k}=\tilde{A}_{i j k} Y_{i j k}+Y_{i j k} \tilde{A}_{i j k}^{T}+F_{i j k}^{T} B_{j}^{T}+B_{j} F_{i j k}+\mu^{-2} E_{i j} E_{i j}^{T}+\gamma_{i} \bar{a}_{i} W_{i} W_{i}^{T} \\
\Omega_{i j k}=\tilde{A}_{i j k}^{T} X_{i j k}+X_{i j k} \tilde{A}_{i j k}+C_{2}^{T} L_{i j k}^{T}+L_{i j k} C_{2} \\
\quad+C_{1}^{T} C_{1}+\left(\bar{a}_{i} / \gamma_{i}\right) T_{i}^{T} T_{i}+\sum_{\substack{h \in Z \\
h \neq i}} \pi_{i h} X_{h j k}+\sum_{\substack{l \in S \\
l \neq j}} \nu_{j l} X_{i l k}+\sum_{\substack{v \in R \\
v \neq k}} \lambda_{k v}^{i j} X_{i j v}
\end{array}\right.
$$


c) (VBU) uncertainties

$$
\left\{\begin{array}{c}
\Upsilon_{i j k}=\tilde{A}_{i j k} Y_{i j k}+Y_{i j k} \tilde{A}_{i j k}^{T}+F_{i j k}^{T} B_{j}^{T}+B_{j} F_{i j k}+\mu^{-2} E_{i j} E_{i j}^{T}+\gamma_{i} I \\
\Omega_{i j k}=\tilde{A}_{i j k}^{T} X_{i j k}+X_{i j k} \tilde{A}_{i j k}+C_{2}^{T} L_{i j k}^{T}+L_{i j k} C_{2} \\
\quad+C_{1}^{T} C_{1}+\left(n / \gamma_{i}\right) \operatorname{diag}\left(M_{i}^{T} M_{i}\right)+\sum_{\substack{h \in Z \\
h \neq i}} \pi_{i h} X_{h j k}+\sum_{\substack{l \in S \\
l \neq j}} \nu_{j l} X_{i l k}+\sum_{\substack{v \in R \\
v \neq k}} \lambda_{k v}^{i j} X_{i j v}
\end{array}\right.
$$

have feasible solutions $X_{i j k}=X_{i j k}^{T}, Y_{i j k}=Y_{i j k}^{T}, L_{i j k}, F_{i j k}$ and $\gamma_{i}>0, \forall i \in Z, j \in S$ and $k \in R$. The corresponding compensator $\left(\varphi_{c}\right)$ is given by

$$
\begin{aligned}
& B_{c i j k}=\left(Y_{i j k}^{-1}-X_{i j k}\right)^{-1} L_{i j k}, \quad C_{c i j k}=F_{i j k} Y_{i j k}^{-1} \\
& A_{c i j k}=\left(X_{i j k}-Y_{i j k}^{-1}\right)^{-1}\left[\tilde{A}_{i j k}^{T}+X_{i j k} \tilde{A}_{i j k} Y_{i j k}+X_{i j k} B_{j} F_{i j k}+L_{i j k} C_{2} Y_{i j k}+\left(\sum_{\substack{h \in Z \\
h \neq i}} \pi_{i h} Y_{h j k}^{-1}\right.\right. \\
& \left.+\sum_{\substack{l \in S \\
l \neq j}} \nu_{j l} Y_{i l k}^{-1}+\sum_{\substack{v \in R \\
v \neq k}} \lambda_{k v}^{i j} Y_{i j v}^{-1}+\Sigma_{i j k}\right) Y_{i j k}+\Phi_{i j k} \\
& \left.+C_{1}^{T}\left(C_{1} Y_{i j k}+D_{1 j} F_{i j k}\right)+\mu^{-2}\left(X_{i j k} E_{i j}+L_{i j k} D_{2 i j}\right)\right] Y_{i j k}^{-1}+0.5 I\left(\sum_{\substack{h \in Z \\
h \neq i}} \pi_{i h}+\sum_{\substack{l \in S \\
l \neq j}} \nu_{j l}+\sum_{\substack{v \in R \\
v \neq k}} \lambda_{k v}^{i j}\right)
\end{aligned}
$$

\section{Conclusion}

In this paper, we have first considered the problematic of dynamic output feedback stochastic stabilization and robust stochastic stabilization of an uncertain AFTCSMP. It was shown that the necessary and sufficient conditions for the internal exponential stability in the mean square sense (robust internal exponential stability, respectively) can be written in terms of an LMI feasibility problem. This leads to an LMI characterization of all dynamical output feedback compensators that satisfied the stability requirements. Having obtained these results, we have moved on the control problem. Indeed, we have introduced an LMI approach to the $\mathcal{H}_{\infty}$ and robust $\mathcal{H}_{\infty}$ control for linear uncertain continuous time AFTCSMP under a dynamic output feedback control. We have derived some linear matrix inequalities whose solutions indicate the achievability of the desired control problems; i.e. we have shown that the $\mathcal{H}_{\infty}$ and robust $\mathcal{H}_{\infty}$ control problematic can be recast as a convex optimization problem under constraints of LMIs which can be solved effectively using the recently developed LMI tool. Then, based on these LMIs, we have given a simple procedure to construct the required output feedback stabilizing controllers.

\section{References}

S. Aberkane, J.C. Ponsart and D. Sauter, Output Feedback Stochastic Stabilization of Active Fault Tolerant Control Systems: LMI Formulation, 16th IFAC World Congress, Prague, Czech Republic, 2005.

M. Blanke, M. Kinnaert and M. Stroswiecki, Diagnosis and Fault-Tolerant Control, Springer, 2003.

E. K. Boukas, P. Shi and K. Benjelloun, On Stabilization of Uncertain Linear Systems with Jump Parameters, International Journal of Control, Vol 72, No. 9, 1999, pp 842-850. 
E. K. Boukas, P. Shi, M. Karan and C. Y. Kaya, Linear Discrete-time Systems with Markovian Jumps and Mode Dependent Time-delay: Stability and Stabilizability, Mathematical Problems in Engineering, Vol.8, No.2, pp.123-133, 2002.

E. K. Boukas and N. F. Al-Muthairi, $\mathcal{H}_{\infty}$ Constant Gain State Feedback of Stochastic Hybrid Systems with Wienner Process, Mathematical Problems in Engineering, Vol.4, pp.333-345, 2004.

E. K. Boukas, Stabilization of Stochastic Nonlinear Hybrid Systems, Int. J. Innovative Computing, Information and Control, Vol.1, No.1, pp.131-141, 2005.

E. K. Boukas, Static Output Feedback Control for Stochastic Hybrid Systems: LMI Approach, Automatica, Vol.42, pp.183-188, 2006.

S. Boyd, L. El Ghaoui, E. Feron and V. Balakrishnan, Linear Matrix Inequalities in System and Control Theory, Philadelphia: SIAM; 1994.

O. L. V. Costa, J. B. R. do Val and J. C. Geromel, Continuous-time state-feedback $\mathcal{H}_{2}$-control of Markovian jump linear systems via convex analysis, Automatica, Vol 35, 1999, pp 259-268.

D. P. de Farias, J. C. Geromel, J. B. R. do Val and O. L. V. Costa, Output Feedback Control of Markov Jump Linear Systems in Continuous-Time, IEEE Transactions on Automatic Control, Vol 45, 2000, pp 944-949.

C. E. de Souza and M. D. Fragoso, $\mathcal{H}_{\infty}$ Control For Linear Systems With Markovian Jumping Parameters, Control Theory and Advanced Technology, Vol 9, No. 2, 1993, pp 457-466.

Y. Ji and H. J. Chizeck, Controllability, stabilizability, and continuous-time Markovian jump linear quadratic control, IEEE Transactions on Automatic Control, Vol 35, 1990, pp 777-788.

Y. Ji and H. J. Chizeck, Jump linear quadratic Gaussian control in continuous time, IEEE Transactions on Automatic Control, Vol 37, 1992, pp 1884-1892.

M. Mahmoud, J. Jiang and Y. Zhang, Analysis of the Stochastic Stability for Active Fault Tolerant Control Systems, Proceedings of the $38^{t h}$ IEEE Conference on Decision \& Control, Phoenix, Arizona USA, 1999, pp 3188-3193.

M. Mahmoud, J. Jiang and Y. Zhang, Optimal Control Law for Fault Tolerant Control Systems, Proceedings of the $39^{\text {th }}$ IEEE Conference on Decision $\&$ Control, Sydney, Australia, 2000, pp 4126-4131.

M. Mahmoud, J. Jiang and Y. Zhang, Stochastic Stability of Fault Tolerant Control Systems with Model Uncertainties, Proceedings of the American Control Conference, Chicago, Illinois, 2000, pp 4284-4288.

M. Mahmoud, J. Jiang and Y. Zhang, Stochastic Stability Analysis of Active Fault-Tolerant Control Systems in the Presence of Noise, IEEE Transactions on Automatic Control, Vol 46, 2001, pp 1810-1815.

M. Mahmoud, J. Jiang and Y. Zhang, Stability of Fault Tolerant Control Systems Driven By Actuators With Saturation, $15^{\text {th }}$ Triennial IFAC World Congress, Barcelona, Spain, 2002, pp 1810-1815.

M. Mahmoud, J. Jiang and Y. Zhang, Active Fault Tolerant Control Systems: Stochastic Analysis and Synthesis, Springer, 2003.

I. R. Petersen, A Stabilzation Algorithm for a Class of Uncertain Linear Systems, Systems and Control Letters, Vol 8, 1987, pp 351-357.

P. Shi and E. K. Boukas, $\mathcal{H}_{\infty}$-Control for Markovian Jumping Linear Systems with Parametric Uncertainty, Journal of Optimization Theory and Applications, Vol 95, 1997, pp 75-99.

P. Shi, E. K. Boukas, S. K. Nguang and X. Guo, Robust disturbance attenuation for discrete-time active fault tolerant control systems with uncertainties, Optimal Control Applications and Methods, Vol 24, 2003, pp 85-101.

R. Srichander and B. K. Walker, Stochastic stability analysis for continuous-time fault tolerant control systems, International Journal of Control, Vol 57, 1993, pp 433-452.

Y. Zhang and J. Jiang, Bibliographical review on reconfigurable fault-tolerant control systems, IFAC SAFEPROCESS, 2003, pp 265-276.

K. Zhou, J. Doyle and K. Glover, Robust and Optimal Control, Prentice Hall, 1996. 